\title{
Cow-baited tents are highly effective in sampling diverse Anopheles malaria vectors in Cambodia
}

\author{
Brandyce St. Laurent ${ }^{1 *} \mathbb{D}$, Kolthida Oy² ${ }^{2}$ Becky Miller ${ }^{1}$, Elizabeth B. Gasteiger ${ }^{1}$, Eunjae Lee ${ }^{1}$, Siv Sovannaroth², \\ Robert W. Gwadz' ${ }^{1}$ Jennifer M. Anderson ${ }^{1}$ and Rick M. Fairhurst ${ }^{1 *}$
}

\begin{abstract}
Background: The accurate monitoring and evaluation of malaria vectors requires efficient sampling. The objective of this study was to compare methods for sampling outdoor-biting Anopheles mosquitoes in Cambodia.

Methods: In the Cambodian provinces of Pursat, Preah Vihear, and Ratanakiri, six different mosquito trapping methods were evaluated: human landing collection (HLC), human-baited tent (HBT), cow-baited tent (CBT), CDC miniature light trap (LT), CDC miniature light trap baited with molasses and yeast (LT-M), and barrier fence (F) in a Latin square design during four or six consecutive nights at the height of the malaria transmission season.

Results: Using all traps, a total of 507, 1175, and 615 anophelines were collected in Pursat, Preah Vihear, and Ratanakiri, respectively. CBTs captured 10- to 20-fold more anophelines per night than the other five sampling methods. All 2297 Anopheles mosquitoes were morphologically identified and molecularly typed using standard morphological keys and sequencing the rDNA ITS2 region to distinguish cryptic species, respectively. Overall, an extremely diverse set of 27 known Anopheles species was sampled. CBTs captured the same molecular species that HLCs and the other four traps did, as well as additional species. Nine specimens representing five Anopheles species (Anopheles hyrcanus, Anopheles barbirostris sensu stricto, Anopheles barbirostris clade III, Anopheles nivipes, and Anopheles peditaeniatus) were infected with Plasmodium falciparum and were exclusively captured in CBTs.

Conclusions: These data indicate that cow-baited tents are highly effective in sampling diverse Anopheles malaria vectors in Cambodia. This sampling method captured high numbers of anophelines with limited sampling effort and greatly reduced human exposure to mosquito bites compared to the gold-standard human landing collection.
\end{abstract}

Keywords: Anopheles, Cambodia, Trap, Outdoor transmission, Malaria, Vector

\section{Background}

Malaria transmission in the Greater Mekong Subregion (GMS), where artemisinin-resistant Plasmodium falciparum parasites have emerged, endangers global malaria control efforts. As treatment failure rates for frontline anti-malarial drugs continue to worsen [1, 2], control efforts focusing on malaria vectors in the GMS have become increasingly important. Western Cambodia

\footnotetext{
*Correspondence: brandyce.stlaurent@nih.gov; rfairhurst@niaid.nih.gov ${ }^{1}$ Laboratory of Malaria and Vector Research, National Institute of Allergy and Infectious Diseases, National Institutes of Health, Rockville, MD 20852, USA

Full list of author information is available at the end of the article
}

in particular has been a hotspot for the evolution and spread of drug-resistant $P$. falciparum parasites. Since these parasites can infect extremely diverse Anopheles species, including the major vector of sub-Saharan Africa, Anopheles coluzzii (formerly Anopheles gambiae $\mathrm{M}$ form) [3], local control and elimination efforts are needed to prevent the spread of these dangerous pathogens to other regions. The natural transmission of these parasites and the efficacy of integrated malaria control efforts in Southeast Asia cannot be characterized without the appropriate sampling of local vectors.

The anopheline vectors in the GMS are incredibly diverse and primarily bite outdoors [4-6], enabling them 
to avoid commonly used vector control interventions, such as indoor residual sprays or insecticide-treated bed nets. While many different traps have been extensively evaluated in regions of highly-endemic malaria transmission in sub-Saharan Africa, little is known about trapping efficacy in many parts of Southeast Asia.

The human-landing collection (HLC) is considered the best sampling method to estimate human exposure to potentially infectious bites by malaria vectors. However, in many regions where vector density is low or vector species demonstrate generalist host-feeding behaviours, the yields of sampling methods like HLCs are insufficient to adequately sample the vector population. In addition, HLCs are extremely labour-intensive and may expose collectors to potentially infectious bites. Several alternatives to HLC have been explored in regions in sub-Saharan Africa and Asia, typically involving a protected human contained in a larger net or trap [7-9]. Other attractive traps include the Centers for Disease Control and Prevention $(\mathrm{CDC})$ miniature light trap, which attracts mosquitoes with a light and sometimes $\mathrm{CO}_{2}$ bait, which in some cases can yield results comparable to those of the HLC [10]. While not labour-intensive, light traps require a battery and a $\mathrm{CO}_{2}$ or odor source to attract mosquitoes. Passive trapping techniques for outdoor-biting malaria vectors, such as a barrier fence, are currently being explored as monitoring and control tools [11]. Low-cost collection techniques that effectively sample malaria vectors are critical for evaluating transmission.

Although a number of thorough surveys have been conducted in the GMS $[4,12,13]$, many studies have not screened "secondary" vectors for Plasmodium infection, but rather screened only the presumed "primary" vectors: Anopheles dirus, Anopheles minimus, and Anopheles maculatus.

Since sampling efficacy and mosquito species distributions can differ by location, trap efficiency was evaluated in three different Cambodian provinces where clusters of clinical malaria cases had been identified during previous peak transmission seasons.

\section{Methods}

\section{Study sites}

From July to August 2013, mosquitoes were collected in a single village in each of three Cambodian provinces. Sayas Village, Ratanakiri $\left(13^{\circ} 32^{\prime} 51.5^{\prime \prime} \mathrm{N} 107^{\circ} 01^{\prime} 28.4^{\prime \prime} \mathrm{E}\right)$ is an isolated community in the forest. Chean Mok commune, Preah Vihear $\left(13^{\circ} 46^{\prime} 11.3^{\prime \prime} \mathrm{N} 104^{\circ} 55^{\prime} 13.4^{\prime \prime} \mathrm{E}\right)$ is a small community at the base of a mountain. Ankrong Village, Pursat $\left(12^{\circ} 18^{\prime} 46.5^{\prime \prime} \mathrm{N} 103^{\circ} 34^{\prime} 13.4^{\prime \prime} \mathrm{E}\right)$ is a rural farming community approximately $40 \mathrm{~km}$ outside of Pursat town. Villages were selected based on clustering of clinical $P$. falciparum malaria cases during previous transmission seasons.

\section{Mosquito sampling}

Adult mosquitoes were collected using six different outdoor sampling methods concurrently at each site:

- Cow-baited tent (CBT) collections took place from 6 p.m. to 6 a.m. An adult cow was loosely tied to a stake in the ground at the centre of a large tent (Coleman $13 \times 15 \mathrm{ft}$. screened canopy tent). Mosquitoes resting on the interior walls of the tent were collected hourly for $5 \mathrm{~min}$ using mouth aspirators. Humanbaited tent (HBT) collections were done similarly, but the human was protected from mosquito bites using a smaller tent (Eureka! solitaire 1-person tent) inside of the same larger tent.

- Human landing collections (HLC) took place from 6 p.m. to 6 a.m. Trained collectors sat with their legs exposed and collected mosquitoes landing on their skin using a mouth aspirator and a flashlight. Human landing collectors sat outside in a dark area and stored mosquitoes in a paper cup, which was collected hourly. Collectors worked in 6-h shifts, switching out with other collectors at midnight, in accordance with the Cambodian National Center for Parasitology, Entomology, and Malaria Control HLC protocol.

- CDC miniature light traps (LT) (John Hock Co., Gainesville, FL) and the same light traps baited with a mixture of molasses and yeast (LT-M) as a source of $\mathrm{CO}_{2}$ [14] were placed outdoors at sampling locations. The LT contains a battery-powered light and fan. Mosquitoes near the fan are sucked into the trap. In this case, the molasses and yeast release $\mathrm{CO}_{2}$ as a lure and the unbaited trap acts as a control with only the light serving as the attractant. Mosquitoes were collected from the LTs every morning between 5:30 a.m. and 6 a.m.

- A barrier-screened fence that was not treated with insecticide was erected between the village and the forest. The barrier fences were $20 \mathrm{~m}$ long and $2 \mathrm{~m}$ high. Two collectors simultaneously sampled the inner, village-facing side of the fence (F-I), and the outer, forest-facing side of the fence (F-O) in a single pass every hour from 6 p.m. to 6 a.m. In Ratanakiri, collections from both sides of the fence were pooled (F).

Three locally representative sites per village were selected for the experiment and sets of traps were rotated through these locations at least $30 \mathrm{~m}$ apart over consecutive nights. Trap locations at least $15 \mathrm{~m}$ from other host attractive cues were selected. An LT and LT-M remained at all three trapping locations each night of collection. HLCs, CBTs, and HBTs were rotated through the three 
locations every night at least $10 \mathrm{~m}$ from either LT. Individual mosquitoes were held separately in paper cups according to collection method and hour, and then morphologically identified in the field. Mosquitoes were stored individually in barcoded $1.5-\mathrm{ml}$ tubes with silica gel desiccant until they were molecularly analysed.

\section{Mosquito identification and molecular analyses}

Genomic DNA was isolated from individual mosquito heads and thoraces using a CTAB-based DNA extraction method. The ribosomal DNA internal transcribed spacer region (rDNA ITS2) was PCR-amplified using ITS2A and ITS2B primers [15] that were developed to differentiate Anopheles cryptic species, and then sequenced. PCR products were visualized on a $1 \%$ agarose gel and purified by mixing $8 \mu \mathrm{l}$ of PCR product with $2 \mathrm{U}$ of exonuclease 1 (USB Corporation, Cleveland, $\mathrm{OH}$ ), $1 \mathrm{U}$ of shrimp alkaline phosphatase (USB Corporation), and $1.8 \mu \mathrm{l}$ of $\mathrm{ddH}_{2} \mathrm{O}$. This cleanup mixture was incubated at $37{ }^{\circ} \mathrm{C}$ for $15 \mathrm{~min}$, and then at $80{ }^{\circ} \mathrm{C}$ for $15 \mathrm{~min}$ to inactivate the enzymes. PCR products were sequenced directly using Sanger sequencing on an ABI 3730 xl DNA analyzer platform (PE Applied Biosystems, Warrington, England). Clean ITS2 sequences for each specimen were blasted using BLASTn against the NCBI GenBank nr database to confirm molecular species identification when compared to voucher and published sequences.

Extracted DNA from each individual mosquito head and thorax was used to test for Plasmodium infection using a nested PCR to amplify a portion of the Plasmodium mitochondrial cytochrome B gene (cytb) [16]. The Sanger sequences of these PCR-positive amplicons were assigned to Plasmodium species by comparing them to known Plasmodium voucher cytb sequences in the NCBI database.

Abdomens of mosquitoes that were blood-fed were separated from the head and thorax, and analysed using a multiplex, blood meal-diagnostic PCR assay based on vertebrate mitochondrial cytb DNA sequences [17]. Blood meal DNA samples that did not amplify in the diagnostic PCR assay were sequenced and blasted against the NCBI GenBank nr database to identify the source of the blood meal.

\section{Data analysis}

Data from each mosquito collection were analysed using GraphPad Prism software version 6 (GraphPad, San Diego, $\mathrm{CA})$. The mean catch differences between sampling methods at each site were analysed using ANOVA. The catches were treated as the dependent variable and compared using ANOVA with a significance threshold of $\mathrm{p} \leq 0.05$. Trap yields were compared only within the same village. The null hypothesis was that there was no difference in nightly anopheline catch between sampling methods (no trap effect). A post-hoc Tukey's HSD test was performed to identify statistically significant differences between total catch due to trap and location effects in the experiment. This analysis was performed on the total number of anophelines captured per night, which was greater than the total number of molecularly analysed anophelines.

\section{Results}

Six different trapping techniques were compared to determine which methods would be appropriate for monitoring outdoor-biting malaria vectors in Cambodia during the peak transmission season. The trapping techniques analysed included cow-baited tents (CBT), human-baited tents (HBT), human landing collections (HLC), CDC miniature light traps (LT), CDC miniature light traps baited with molasses and yeast (LT-M), and a barrier fence (F) with collections on the village-facing (F-I) and forest-facing (F-O) sides of the fence.

Overall, 27 known Anopheles species were collected in three provinces (Table 1). These species represent ten diverse species groups, including four species in the Anopheles annularis group, five species in the Anopheles barbirostris group, four species in the Anopheles funestus group, six species in the Anopheles hyrcanus group, three species in the $A n$. maculatus group, as well as An. dirus A, Anopheles kochi, Anopheles splendidus, Anopheles tessellatus, and Anopheles vagus. These findings indicate an extreme level of Anopheles diversity, even when compared to other areas in the GMS. Many of the molecularly identified species collected were only recently recognized as distinct subspecies, as anopheline species complexes in this region are being more accurately described [18], including several members of the An. barbirostris [19], An. annularis [20], and An. hyrcanus groups [21, 22].

The five most prevalent species across the three sitesAnopheles nivipes ( $\mathrm{n}=601)$, An. vagus ( $\mathrm{n}=564), A n$. kochi $(\mathrm{n}=208)$, and two members of the An. barbirostris complex, An. barbirostris $(\mathrm{n}=145)$ and Anopheles saeungae $(\mathrm{n}=157)$-represented $73 \%$ of the total collection (Table 1). These were the most common species collected by most of the trapping methods, indicating that they were likely the most abundant species in each sampling site during the sampling period. Anopheles nivipes, $A n$. vagus, and An. saeungae were collected in all trap types (Table 2), with 88, 91 and $94 \%$ of them collected in the CBT, respectively. Anopheles kochi were collected in the CBT, HBT, HLC, F-I, and LT-M. Anopheles barbirostris (clade III) were predominantly collected in the CBT (140/145, $97 \%)$, while only a few specimens were sampled in the HBT $(n=1)$ and HLC $(n=4)$. These five most-prevalent species displayed different biting patterns in the three collection sites. Anopheles vagus 
Table 1 Molecular identification of 2297 Anopheles species according to Cambodian province

\begin{tabular}{|c|c|c|c|c|}
\hline Molecular identification & Pursat & Preah Vihear & Ratanakiri & Total \\
\hline \multicolumn{5}{|l|}{ Annularis group } \\
\hline An. annularis & & 1 & 7 & 8 \\
\hline An. nivipes & 106 & 276 & 219 & 601 \\
\hline An. pallidus & 1 & 1 & & 2 \\
\hline An. philippinensis & 47 & 51 & 80 & 178 \\
\hline \multicolumn{5}{|l|}{ Barbirostris group } \\
\hline An. barbirostris & 1 & 3 & 4 & 8 \\
\hline $\begin{array}{l}\text { An. barbirostris (barbirostris } \\
\text { clade III) }\end{array}$ & 17 & 77 & 51 & 145 \\
\hline An. campestris & & 1 & & 1 \\
\hline $\begin{array}{l}\text { An. saeungae (barbirostris } \\
\text { clade IV) }\end{array}$ & 7 & 71 & 79 & 157 \\
\hline An. wejchoochotei & & 12 & 1 & 13 \\
\hline \multicolumn{5}{|l|}{ An. dirus complex } \\
\hline An. $\operatorname{dirus} A$ & 2 & 23 & 4 & 29 \\
\hline \multicolumn{5}{|l|}{ Funestus group } \\
\hline An. aconitus & & 13 & 4 & 17 \\
\hline An. minimus $A$ & & 5 & 2 & 7 \\
\hline An.jeyporiensis & 1 & & 3 & 4 \\
\hline An.pampanai & 3 & & 6 & 9 \\
\hline \multicolumn{5}{|l|}{ Hyrcanus group } \\
\hline An. argyropus & & & 7 & 7 \\
\hline An. crawfordi & 85 & & 1 & 86 \\
\hline An. hyrcanus & 1 & & 1 & 2 \\
\hline An. peditaeniatus & 4 & 7 & 21 & 32 \\
\hline An. nigerrimus & & & 20 & 20 \\
\hline An. nitidus & 2 & & 1 & 3 \\
\hline \multicolumn{5}{|l|}{ Kochi group } \\
\hline An. kochi & 72 & 130 & 6 & 208 \\
\hline \multicolumn{5}{|l|}{ Maculatus group } \\
\hline An. sawadwongporni & 20 & 19 & 4 & 43 \\
\hline An. rampae & 2 & 29 & 18 & 49 \\
\hline An. karwari & 13 & 12 & 7 & 32 \\
\hline \multicolumn{5}{|l|}{ Other } \\
\hline An. splendidus & & 8 & 7 & 15 \\
\hline An. tessellatus & 8 & 32 & 13 & 53 \\
\hline An.vagus & 111 & 404 & 49 & 564 \\
\hline Unknown Anopheles & 4 & & & 4 \\
\hline Total & 507 & 1175 & 615 & 2297 \\
\hline
\end{tabular}

The numbers of Anopheles species, identified by rDNA ITS2 sequences, collected during a short period in Pursat, Preah Vihear, and Ratanakiri, Cambodia are shown. Members of common cryptic species complexes are arranged by their corresponding species group

had peak biting activity early in the night in Pursat and Preah Vihear, but was less prevalent and had no apparent peak biting period in Ratanakiri (Fig. 1). Anopheles nivipes appeared to be actively biting later in the night, and in gradually increasing numbers after 10 p.m.
Anopheles saeungae was abundant in the first half of the night in Preah Vihear and Ratanakiri, but was not prevalent in Pursat. Anopheles kochi and Anopheles barbirostris seemed to bite throughout the night, though further sampling is necessary to more accurately evaluate their biting behaviours.

Anopheles dirus, considered the major vector species in the GMS $[6,23]$, represented only $1 \%$ of the total collection, and were collected in the CBT ( $\mathrm{n}=12$, two sites), HBT ( $\mathrm{n}=10$, two sites), HLC ( $\mathrm{n}=6$, two sites), and F-O $(\mathrm{n}=1$, one site) (Table 2). Culex, Aedes, Toxorhynchites, and Mansonia were also present in these collections, particularly in LT collections.

The method of sampling, but neither the night of collection nor the location of traps, significantly affected the number of anophelines captured in all three provinces. The CBT yielded the highest total catch and Anopheles species richness in each province. A total of 2297 anopheline mosquitoes were collected using all trap methods in all three provinces. The CBT collected $90 \%(n=2055)$ of the total anophelines, while the HBT, HLC, F-I, F-O, LT, and LT-M collected only $4 \%(\mathrm{n}=98), 3 \%(\mathrm{n}=72), 1 \%$ $(\mathrm{n}=19),<1 \%(\mathrm{n}=5), 1 \%(\mathrm{n}=15)$, and $1 \%(\mathrm{n}=14)$ of the total anophelines, respectively.

Twenty Anopheles species were collected over six nights in Angkrong Village, Pursat (Table 1), where trap method significantly affected capture rates $(\mathrm{F}=33.6$; $\mathrm{df}=6,30 ; \mathrm{p}<0.0001$, ANOVA) (Fig. 2). The CBT trapped significantly more anophelines per night than the other six methods ( $\mathrm{p} \leq 0.0001$, Tukey's HSD test). The HLC captured significantly more anophelines per night than the F-I and F-O ( $\mathrm{p} \leq 0.05)$, and LT and LT-M ( $\leq 0.01)$.

Nineteen Anopheles species were collected over six nights in Chean Mok Pagoda Village, Preah Vihear (Table 1), where trap method also significantly affected capture rates $(\mathrm{F}=61.6 ; \mathrm{df}=6,30 ; \mathrm{p}<0.0001)$ (Fig. 2). The CBT trapped significantly more anophelines per night than all other methods ( $\mathrm{p} \leq 0.0001)$. The HBT and HLC captured similar numbers of anophelines per night, and showed a non-significant trend in trapping about four-fold more anophelines per night than the F-I, F-O, LT, and LT-M.

An extremely diverse set of 25 Anopheles species was collected over only four nights (due to extreme weather conditions) in Sayas Village, Ratanakiri (Table 1), where trap method also significantly affected capture rates $(\mathrm{F}=19.71 ; \mathrm{df}=5,15 ; \mathrm{p} \leq 0.0001)$ (Fig. 2). The CBT trapped significantly more anophelines per night than all other methods ( $\mathrm{p} \leq 0.0001)$. The HBT and HLC captured similar numbers of anophelines per night, and showed a non-significant trend in trapping about fivefold more anophelines per night than the F-I, F-O, LT, and LT-M. 


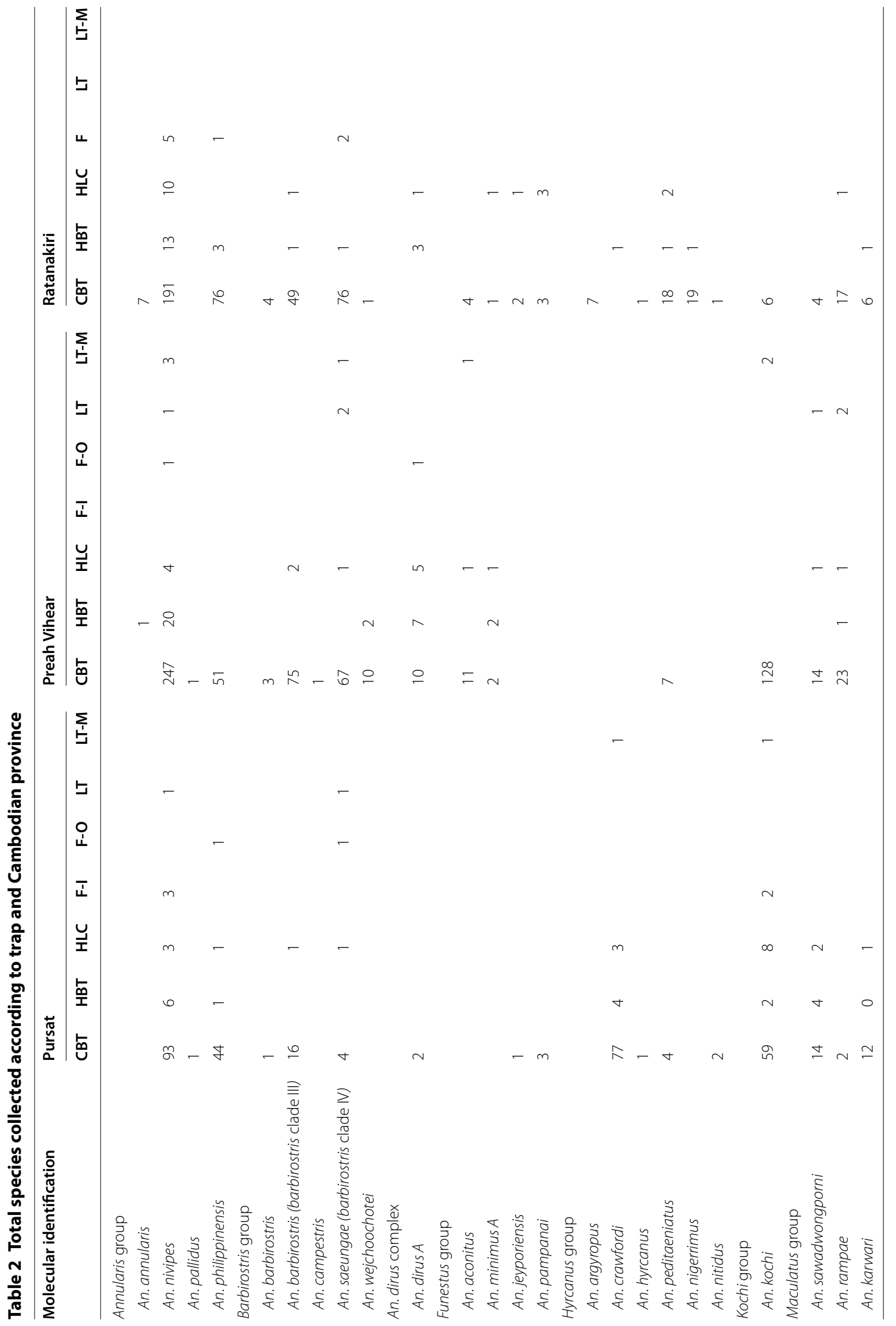




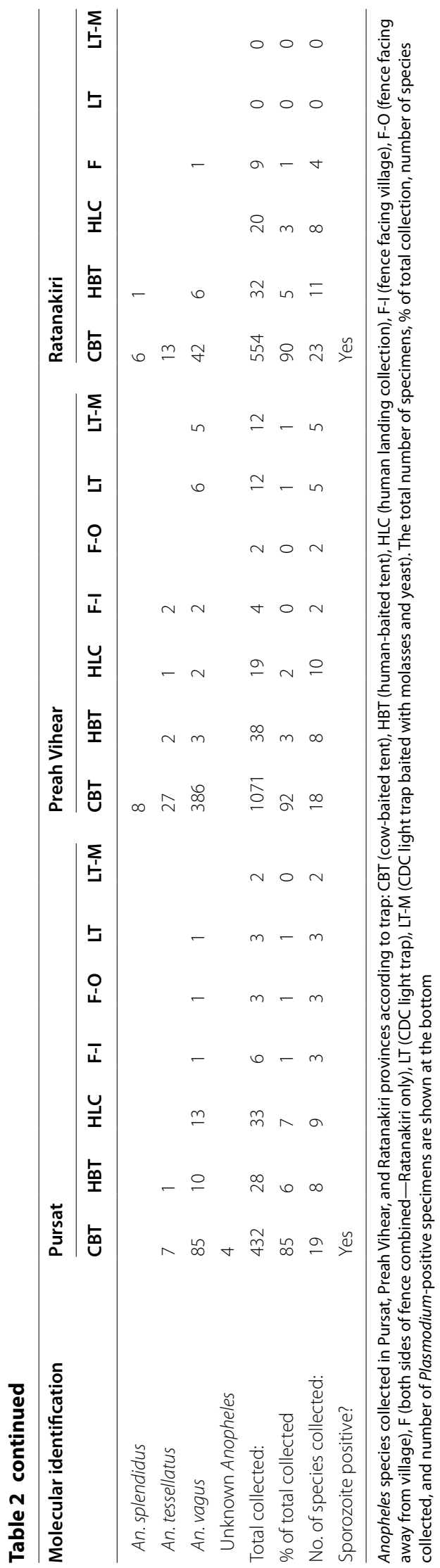




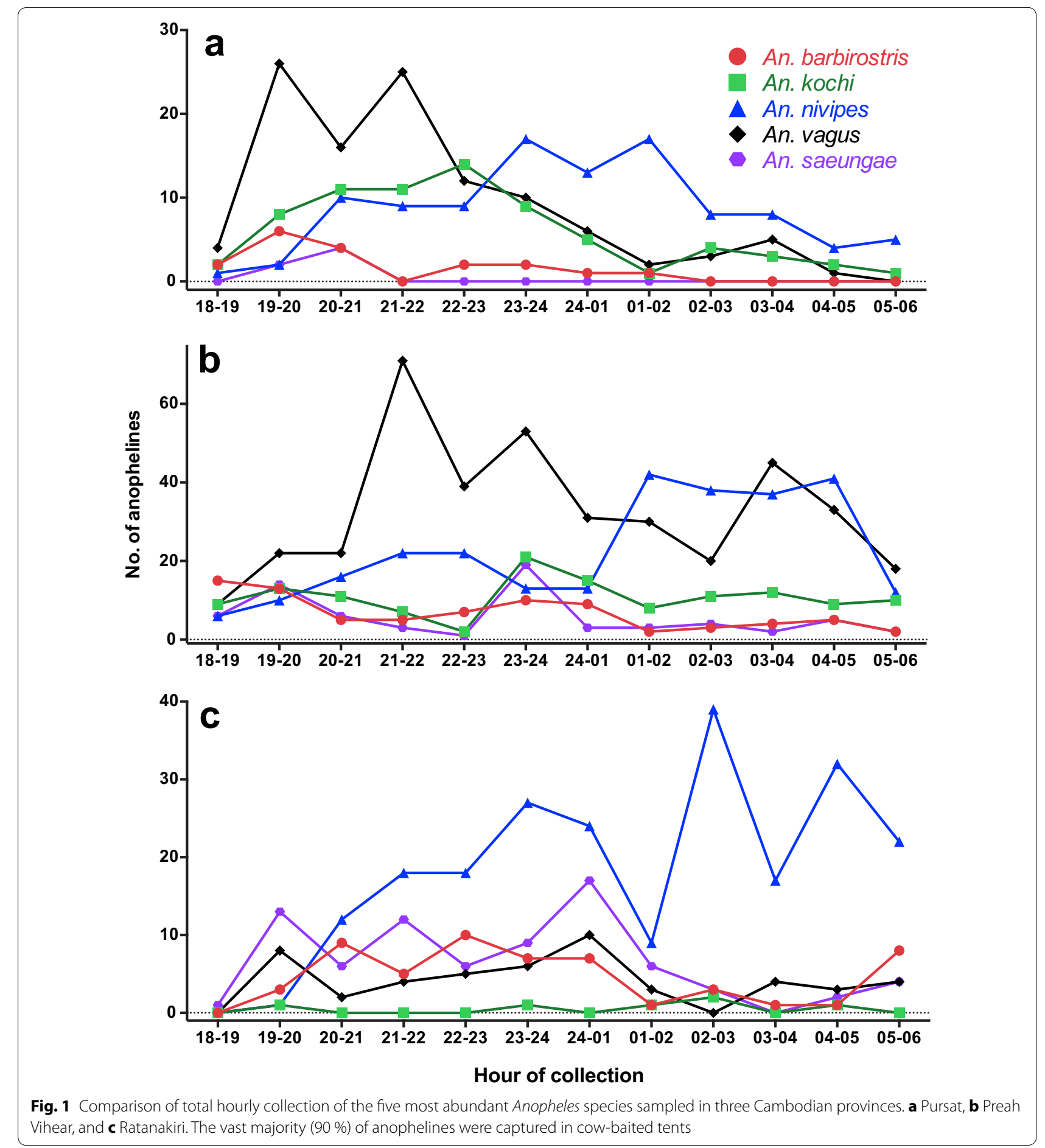

The species distributions between the three sites were relatively consistent (Tables 1,2), but there were some exceptions. For example, only a single representative of the An. hyrcanus group, Anopheles peditaeniatus, was collected in Preah Vihear, while 3 and 5 other members of this group were collected in Pursat and Ratanakiri, respectively. Also, while distinct members of the $A n$. funestus group were collected in Pursat and Preah Vihear, all of them were represented in Ratanakiri. Ongoing longitudinal collections at these sites will likely resolve these apparent differences in species distribution with more sampling points over time (Table 1). 


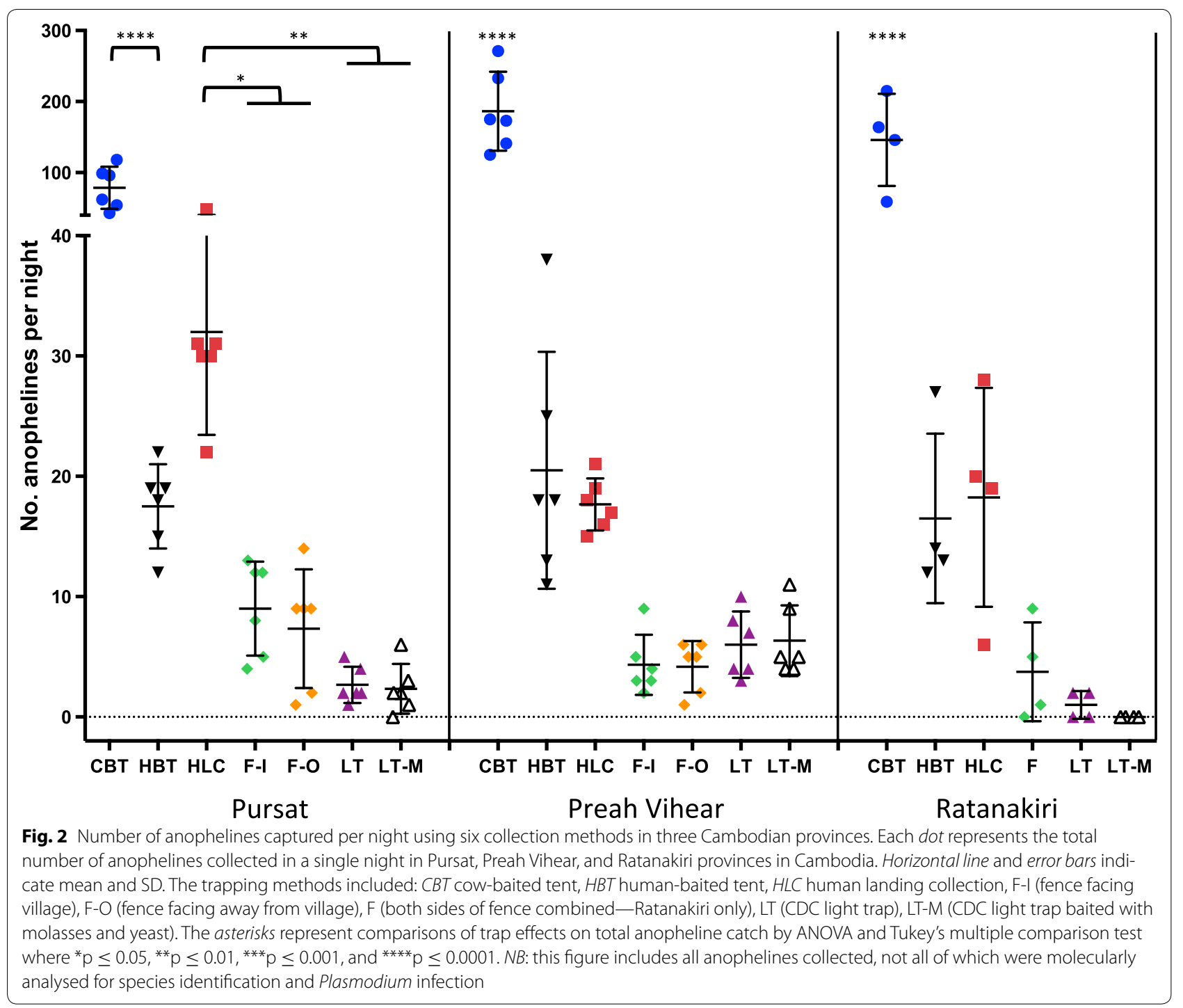

Of the 206, 664, and 439 individual mosquito blood meals typed from collections in Pursat, Preah Vihear, and Ratanakiri, more than $97 \%$ were from cow, $1.5 \%$ were from cow and another host (e.g., human, goat, or pig), and $0.8 \%$ were from human only. Over $99 \%$ of Anopheles mosquitoes collected in the CBT were blood-fed on a cow (see Additional file 1). Each of the 27 species captured, including An. dirus, were found to be bloodfed on a cow.

All 2297 molecularly-identified specimens (Table 1) were analysed for Plasmodium infection by PCR [16]. Nine $(0.4 \%)$ of these specimens representing five distinct Anopheles species were positive for P. falciparum (Table 3), and all were captured in the CBT. These positive specimens included one An. hyrcanus in Pursat, and one An. barbirostris, two An. barbirostris (clade III) (a newly described cryptic species [19]), one An. hyrcanus, three An. nivipes, and one An. peditaeniatus in Ratanakiri; no positive specimens were found in Preah Vihear. Both of the An. hyrcanus specimens collected in this study (one from Pursat, one from Ratanakiri) were positive for $P$. falciparum. The infection rates of the other four species were 1.4, 3.9, 4.8, and $25 \%$ (Table 3). Further sampling and analysis of a greater number of field-collected female anophelines are needed to determine the natural infection rates of these and other species.

\section{Discussion}

This study demonstrated that CBTs attract much higher numbers of anophelines than HLCs or HBTs, capturing hundreds of Anopheles mosquitoes in a single night without risking human exposure to potentially infectious 
Table 3 Individual Plasmodium falciparum-positive Anopheles specimens

\begin{tabular}{|c|c|c|c|c|}
\hline $\begin{array}{l}\text { Molecular } \\
\text { species }\end{array}$ & $\begin{array}{l}\text { Morphological } \\
\text { species }\end{array}$ & $\begin{array}{l}\text { Positive/ } \\
\text { total }\end{array}$ & Province & Hour \\
\hline An. hyrcanus & An. hyrcanus gr. & $1 / 1$ & Pursat & 8-9 p.m. \\
\hline An. barbirostris s.s. & An. barbirostris & $1 / 4$ & Ratanakiri & 7-8 p.m. \\
\hline $\begin{array}{l}\text { An. barbirostris } \\
\text { clade III }\end{array}$ & An. barbirostris & $2 / 51$ & Ratanakiri & 5-6 a.m. \\
\hline $\begin{array}{l}\text { An. barbirostris } \\
\text { clade III }\end{array}$ & An. barbirostris & & & \\
\hline An. hyrcanus s.s. & An. hyrcanus & $1 / 1$ & Ratanakiri & 4-5 a.m. \\
\hline An. nivipes & An. philippinensis & $3 / 219$ & Ratanakiri & 2-3 a.m. \\
\hline An. nivipes & An. philippinensis & & & 4-5 a.m. \\
\hline An. nivipes & An. philippinensis & & & 4-5 a.m. \\
\hline An. peditaeniatus & An. nigerrimus & $1 / 21$ & Ratanakiri & 10-11 p.m. \\
\hline
\end{tabular}

Molecular and morphological species identification, total positive for Plasmodium falciparum over the number of that species tested for $P$. falciparum infection by PCR, province of collection, and hour of collection are shown. All $P$. falciparum-positive specimens were collected in cow-baited tents

bites, as do standard HLCs. During a short collection period in only three villages across Cambodia, CBTs captured a broad range of anopheline species that are known to be human-biting in Cambodia as well as many that are considered animal-biting or catholic in their feeding habits. In the GMS, the low HLC sampling rates observed in this and other studies do not justify the use of HLC as a regular sampling and monitoring tool. There are some indications that providing prophylaxis to collectors during and immediately following collection periods reduces malaria incidence [24], when HLC is the most effective means of collecting vectors. However, prophylaxis does not prevent other common vector-borne diseases in the GMS, such as Japanese encephalitis, dengue, and filariasis. Alternatives to HLC are needed in Southeast Asia to mitigate the risk of volunteer exposure to potentiallyinfectious bites and to efficiently and cost-effectively monitor malaria vectors.

Light traps have been proposed as a more cost-effective way to collect mosquitoes. In many regions, however, LTs have been found to sample a different species distribution than HLCs, while also catching far fewer mosquitoes $[25,26]$, and the efficacy of LT captures have been shown to vary by location $[27,28]$. In this study, LTs caught very low numbers of anophelines in locations where hundreds of other anopheline mosquitoes were caught per night.

While all of the Anopheles species collected in each of the trapping methods evaluated were also collected in the CBT, four additional (Anopheles pallidus, An. barbirostris, An. hyrcanus, and Anopheles nitidus) and one unknown Anopheles species were collected only in the CBT. This finding is useful because it suggests that the CBT will enable entomologists to screen higher numbers of a broader range of Anopheles species for Plasmodium infection than with any other sampling method tested here. The high rate of cow blood-fed mosquitoes was likely due both to the high attractiveness of the cow due to a greater body size and $\mathrm{CO}_{2}$ /odor output, and the availability of the cow for a sustained and successful blood feed. The majority of CBT-captured mosquitoes were bloodfed and $99 \%$ of all of the bloodfed mosquitoes captured in any of the traps were bloodfed on a cow.

The presumed major vector in the GMS, An. dirus A, comprised $<1 \%$ of the total collection and was just as scarce in the HLC and HBT collections. While major seasonal shifts in Anopheles species and vector composition do occur in many localities, it is unlikely that $A n$. dirus is the major vector throughout the year and across different ecotypes in the GMS. The most abundant species captured in this study, An. nivipes, An. vagus, An. kochi, An. barbirostris, and $A n$. saeungae, are all considered to be outdoor-biting and generalist-feeding. Despite the fact that many secondary vectors can be infected with $P$. falciparum and Plasmodium vivax in laboratory and field settings [13, 29-32], these vectors are still not regularly screened for infection. Since ELISA positivity can overestimate entomological inoculation rates [33], PCR analysis of DNA extracted from the head and thorax alone was used, along with sequence data, to more accurately identify Plasmodium-infected anophelines. None of the six distinct $P$. falciparum-infected Anopheles species identified would have been screened for infection in typical surveys, as they belong to species groups that are not considered major vectors in the GMS. Given the low infection rates among many of these species, further field investigations are required to assess various species' roles in malaria transmission in Cambodia. Since all of the Plasmodiuminfected mosquitoes identified were collected in the CBT, this trapping method is likely to be the most informative. Given the diversity and P. falciparum infection of Anopheles species captured using the CBT, interventions utilizing cow baits could be effective for targeting outdoor and generalist-feeding vectors in the GMS to reduce transmission.

The sampling sites selected for this study represent regions with a very complex outdoor transmission system, where more than 20 Anopheles species were present at a single site, and where bed nets and indoor residual sprays may not target GMS vectors that are active and host-seeking when people are outdoors [34]. This sampling effort represents only a single time point in each province during the high malaria transmission season. Efforts to comprehensively evaluate the seasonal and spatial distribution of malaria vector species over an entire year in Cambodia are underway.

Malaria elimination in the GMS will require sampling methods that screen all potential vector species, 
particularly those vectors that bite and rest outdoors where transmission is occurring. The effective and unbiased evaluation of diverse vectors in this region will be critical for the containment of multidrug-resistant parasites emerging and spreading from Cambodia.

\section{Conclusions}

This study shows that cow-baited tents were able to capture high numbers of similar and diverse Anopheles species when compared to human landing collections and other commonly used collection methods, in three distinct Cambodian provinces. In the Greater Mekong Subregion, where malaria transmission occurs outdoors, this strategy may provide an effective alternative to human landing collections for sampling diverse outdoor malaria vectors.

\section{Additional file}

Additional file 1. Bloodmeal analysis of bloodfed anophelines, according to trap type, province, and species. The data provided represent identification of animal sources of blood meals found in individual Anopheles mosquitoes. These were identified using a multiplex PCR assay, and are grouped according to each mosquito's province of collection and molecular species identification.

\section{Abbreviations}

GMS: Greater Mekong Subregion; CBT: cow-baited tent; HBT: human-baited tent; HLC: human landing collection; F: barrier fence collection; F-l: village (inner)-facing side of the barrier fence; $\mathrm{F}$-O: forest (outer)-facing side of the barrier fence; LT: CDC-miniature light trap; LT-M: CDC-miniature light trap baited with yeast and molasses.

\section{Authors' contributions}

$B S T L, J M A, R M F$, and RG designed the study. BSTL, KO, and SS coordinated field collections in Cambodia. BSTL, BAM, EG, and EL performed the molecular analyses. BSTL and RMF prepared the manuscript. All authors read and approved the final manuscript.

\section{Author details}

${ }^{1}$ Laboratory of Malaria and Vector Research, National Institute of Allergy and Infectious Diseases, National Institutes of Health, Rockville, MD 20852, USA. ${ }^{2}$ National Center for Parasitology, Entomology, and Malaria Control, Phnom Penh 12101, Cambodia.

\section{Acknowledgements}

Thank you to our collaborators at the National Center for Parasitology, Entomology, and Malaria Control (CNM) in Phnom Penh, Cambodia for their support of this work.

\section{Competing interests}

The authors declare that they have no competing interests.

\section{Availability of data}

This article is published Open Access. The datasets generated or analysed during the current study are available from the corresponding authors on reasonable request.

\section{Funding}

This study was funded by the Intramural Research Program, National Institute of Allergy and Infectious Diseases, US National Institutes of Health.

Received: 14 June 2016 Accepted: 10 August 2016

Published online: 30 August 2016
References

1. Ashley EA, Dhorda M, Fairhurst RM, Amaratunga C, Lim P, Suon S, et al. Spread of artemisinin resistance in Plasmodium falciparum malaria. N Engl J Med. 2014;371:411-23.

2. Amaratunga C, Lim P, Suon S, Sreng S, Mao S, Sopha C, et al. Dihydroartemisinin-piperaquine resistance in Plasmodium falciparum malaria in Cambodia: a multisite prospective cohort study. Lancet Infect Dis. 2016;16:357-65.

3. St. Laurent B, Miller B, Burton TA, Amaratunga C, Men S, Sovannaroth S, et al. Artemisinin-resistant Plasmodium falciparum clinical isolates can infect diverse mosquito vectors of Southeast Asia and Africa. Nat Commun. 2015;6:8614.

4. Durnez L, Mao S, Denis L, Roelants P, Sochantha T, Coosemans M. Outdoor malaria transmission in forested villages of Cambodia. Malar J. 2013;12:329.

5. Sanh NH, Van Dung N, Thanh NX, Trung TN, Van Co T, Cooper RD. Forest malaria in central Vietnam. Am J Trop Med Hyg. 2008;79:652-4.

6. Obsomer V, Defourny P, Coosemans M. Predicted distribution of major malaria vectors belonging to the Anopheles dirus complex in Asia: ecological niche and environmental influences. PLoS One. 2012;7:e50475.

7. Krajacich BJ, Slade JR, Mulligan RT, Labrecque B, Kobylinski KC, Gray M, et al. Design and testing of a novel, protective human-baited tent trap for the collection of anthropophilic disease vectors. J Med Entomol. 2014;51:253-63.

8. Tangena JA, Thammavong P, Hiscox A, Lindsay SW, Brey PT. The human-baited double net trap: an alternative to human landing catches for collecting outdoor biting mosquitoes in Lao PDR. PLoS One. 2015;10:e0138735.

9. Govella NJ, Moore JD, Killeen GF. An exposure-free tool for monitoring adult malaria mosquito populations. Am J Trop Med Hyg. 2010;83:596-600.

10. Briet OJ, Huho BJ, Gimnig JE, Bayoh N, Seyoum A, Sikaala CH, et al. Applications and limitations of Centers for Disease Control and Prevention miniature light traps for measuring biting densities of African malaria vector populations: a pooled-analysis of 13 comparisons with human landing catches. Malar J. 2015;14:247.

11. Burkot TR, Russell TL, Reimer LJ, Bugoro H, Beebe NW, Cooper RD, et al. Barrier screens: a method to sample blood-fed and host-seeking exophilic mosquitoes. Malar J. 2013;12:49.

12. Suwonkerd W, Tsuda Y, Overgaard HJ, Chawprom S, Tuno N, Prajakwong $\mathrm{S}$, et al. Changes in malaria vector densities over a 23 year period in Mae Hong Son Province, northern Thailand. Southeast Asian J Trop Med Public Health. 2004;35:316-24.

13. Harbach RE, Gingrich JB, Pang LW. Some entomological observations on malaria transmission in a remote village in northwestern Thailand. DTIC Document; 1987.

14. Mweresa CK, Omusula P, Otieno B, Van Loon JJ, Takken W, Mukabana WR. Molasses as a source of carbon dioxide for attracting the malaria mosquitoes Anopheles gambiae and Anopheles funestus. Malar J. 2014;13:160.

15. Beebe NW, Saul A. Discrimination of all members of the Anopheles punctulatus complex by polymerase chain reaction-restriction fragment length polymorphism analysis. Am J Trop Med Hyg. 1995;53:478-81.

16. Steenkeste N, Incardona S, Chy S, Duval L, Ekala MT, Lim P, et al. Towards high-throughput molecular detection of Plasmodium: new approaches and molecular markers. Malar J. 2009;8:86.

17. Kent RJ, Norris DE. Identification of mammalian blood meals in mosquitoes by a multiplexed polymerase chain reaction targeting cytochrome $\mathrm{B}$. Am J Trop Med Hyg. 2005;73:336-42.

18. Manguin S, Garros C, Dusfour I, Harbach RE, Coosemans M. Bionomics, taxonomy, and distribution of the major malaria vector taxa of Anopheles subgenus Cellia in Southeast Asia: an updated review. Infect Genet Evol. 2008;8:489-503.

19. Paredes-Esquivel C, Donnelly MJ, Harbach RE, Townson H. A molecular phylogeny of mosquitoes in the Anopheles barbirostris subgroup reveals cryptic species: implications for identification of disease vectors. Mol Phylogenet Evol. 2009;50:141-51.

20. Walton C, Somboon P, Harbach RE, Zhang S, Weerasinghe I, O'Loughlin SM, Phompida S, et al. Molecular identification of mosquito species in the Anopheles annularis group in southern Asia. Med Vet Entomol. 2007;21:30-5.

21. Ngo CT, Harbach RE, Garros C, Parzy D, Le HQ, Manguin S. Taxonomic assessment of Anopheles crawfordi and An. dangi of the Hyrcanus group of subgenus Anopheles in Vietnam. Acta Trop. 2013;128:623-9. 
22. Paredes-Esquivel C, Harbach RE, Townson H. Molecular taxonomy of members of the Anopheles hyrcanus group from Thailand and Indonesia. Med Vet Entomol. 2011;25:348-52.

23. O'Loughlin SM, Okabayashi T, Honda M, Kitazoe Y, Kishino H, Somboon P, et al. Complex population history of two Anopheles dirus mosquito species in Southeast Asia suggests the influence of Pleistocene climate change rather than human-mediated effects. J Evol Biol. 2008;21:1555-69.

24. Gimnig JE, Walker ED, Otieno P, Kosgei J, Olang G, Ombok M, et al. Incidence of malaria among mosquito collectors conducting human landing catches in western Kenya. Am J Trop Med Hyg. 2013;88:301-8.

25. Hii JL, Smith T, Mai A, Ibam E, Alpers MP. Comparison between anopheline mosquitoes (Diptera: Culicidae) caught using different methods in a malaria endemic area of Papua New Guinea. Bull Entomol Res. 2000;90:211-9.

26. Sithiprasasna R, Jaichapor B, Chanaimongkol S, Khongtak P, Lealsirivattanakul T, Tiang-Trong S, et al. Evaluation of candidate traps as tools for conducting surveillance for Anopheles mosquitoes in a malaria-endemic area in western Thailand. J Med Entomol. 2004;41:151-7.

27. Gunasekaran K, Jambulingam P, Sadanandane C, Sahu SS, Das PK. Reliability of light trap sampling for Anopheles fluviatilis, a vector of malaria. Acta Trop. 1994;58:1-11.

28. Singh N, Mishra AK. Efficacy of light-traps in sampling malaria vectors in different ecological zones in central India. Southeast Asian J Trop Med Publ Health. 1997;28:196-202.
29. Somboon P, Suwonkerd W, Lines J. Susceptibility of Thai zoophilic Anophelines and suspected malaria vectors to local strains of human malaria parasites. Southeast Asian J Trop Med Public Health. 1994;25:766-70.

30. Coleman RE, Sithiprasasna R, Kankaew P, Kiaattiut C, Ratanawong S, Khuntirat B, et al. Naturally occurring mixed infection of Plasmodium vivax VK210 and P. vivax VK247 in anopheles mosquitoes (Diptera: Culicidae) in western Thailand. J Med Entomol. 2002;39:556-9.

31. Rahman WA. Abu Hassan A, Adanan C, Mohd Razha R. A report of Anopheles (Diptera: Culicidae) attracted to cow bait in a malaria endemic village in Peninsular Malaysia near the Thailand border. Southeast Asian J Trop Med Publ Health. 1995;26:359-63.

32. Thongsahuan S, Baimai V, Junkum A, Saeung A, Min GS, Joshi D, et al. Susceptibility of Anopheles campestris-like and Anopheles barbirostris species complexes to Plasmodium falciparum and Plasmodium vivax in Thailand. Mem Inst Oswaldo Cruz. 2011:106:105-12.

33. Durnez L, Van Bortel W, Denis L, Roelants P, Veracx A, Trung HD, et al. False positive circumsporozoite protein ELISA: a challenge for the estimation of the entomological inoculation rate of malaria and for vector incrimination. Malar J. 2011:10:195.

34. Gryseels C, Durnez L, Gerrets R, Uk S, Suon S, Set S, et al. Re-imagining malaria: heterogeneity of human and mosquito behaviour in relation to residual malaria transmission in Cambodia. Malar J. 2015;14:165.

\section{Submit your next manuscript to BioMed Central and we will help you at every step:}

- We accept pre-submission inquiries

- Our selector tool helps you to find the most relevant journal

- We provide round the clock customer support

- Convenient online submission

- Thorough peer review

- Inclusion in PubMed and all major indexing services

- Maximum visibility for your research

Submit your manuscript at www.biomedcentral.com/submit 